\title{
Eine neue Theorie des Menschen
}

\section{Piet van Spijk}

Dr. med. und Dr. phil., Facharzt für Allgemeine Innere Medizin, Mitglied FMH, Präsident Forum Medizin und Philosophie

1 Whitehead A.N.: Prozess und Realität (besonders: Kapitel X, «Prozess»), 1987. Koutroufinis A.: Prozesse des Lebendigen (Einleitung, S. 9-16), 2007. https://de.wikipedia.org/ wiki/Prozessphilosophie 2 Dieses Wissen kann die Grundlage für eine, in der Medizin wichtigen ethische Haltung schaffen sich an der «Ehrfurcht vor dem Leben» im Sinne Albert Schweitzers anzulehnen.
In einem SÄZ-Artikel mit dem Titel «Die Medizin: Auf der Suche nach einem neuen Menschenbild» kritisiere ich die Tatsache, dass die westliche Medizin mit einem Menschenbild zu arbeiten pflegt, welches nicht mehr dem heutigen Stand der Wissenschaft entspricht [1]. Es geht jetzt darum, eine Alternative, eine neue Theorie des Menschen anzubieten, welche der Medizin als solide Basis in ihrer praktischen Arbeit dienen kann. Am Anfang dieser Theorie steht die folgende Aussage:

«Der Mensch ist das Lebewesen, welches Geschichten erzählt - auch über sich selber.»

Schon Aristoteles verstand den Menschen als ein Lebewesen, welches sich von allen anderen in einer spezifischen Eigenschaft unterscheidet: in seiner Fähigkeit zu sprechen.

Die Theorie hinter der oben stehenden Aussage bricht aber mit einer ebenfalls auf Aristoteles zurückgehenden Tradition: Sie sieht Lebewesen nicht mehr als Dinge oder Substanzen und verlässt die metaphysische Grundposition der sog. Substanzontologie. Sie pflegt neu ein Prozessdenken. Die Welt besteht in dieser prozessphilosophischen Sichtweise nicht mehr aus Dingen, welche in einer mehr oder weniger lockeren Weise

\section{Résumé}

Une représentation claire de l'être humain est requise pour que la médecine puisse à juste titre se qualifier de «médecine humaine». Cet article ébauche une nouvelle théorie de l'être humain, destinée à servir de base solide à la médecine dans son travail pratique. Elle est nécessaire parce que les théories antérieures, qui considèrent l'homme en tant que machine complexe ou qu'unité biopsychosociale, se révèlent de plus en plus dépassées [1].

La nouvelle théorie de l'être humain met en avant sa capacité à parler, qui le distingue de tous les autres êtres vivants. Elle est en outre caractérisée par un changement de paradigme, de l'ontologie des substances (les gens sont vus comme des choses ou des objets) à la philosophie des processus. L'article se poursuit par une brève explication des fondamentaux de la philosophie des processus, s'attachant notamment à montrer ce que signifie voir le monde et l'être humain comme une symphonie presque infinie de processus petits et grands, lents et rapides.

Enfin, il présente les implications de cette théorie pour la médecine du futur.

mit anderen Dingen in Verbindung und Austausch stehen. Sie besteht vielmehr aus einer unendlich komplexen Vielfalt von ineinandergreifenden, schwirrenden und schwingenden Vorgängen oder Prozessen, die mit Gesetzen der Mechanik alleine nicht zu verstehen sind.

\section{Prozesse verstehen}

Wenn wir jetzt von einer Prozess-philosophischen Theorie (PpT) des Menschen sprechen, wird es nötig, dazu einige Erläuterungen zu geben:

1. Lebewesen haben nach diesem Verständnis mit festen Dingen wie Steinen oder Maschinen nichts gemeinsam, sondern lassen sich besser mit Wellen, Wolken oder Flüssen vergleichen. Alles an den Lebewesen, von den einfachsten Bakterien bis hin zum Menschen, ist im Fluss.

2. Ein Wasserfall erscheint von Weitem fälschlicherweise als ein fester Gegenstand. Aus der Nähe betrachtet, ist es ein von Wasser mit hoher Energie durchflossener, auf den Erdmittelpunkt zustrebender Prozess. Die Lebewesen scheinen gegenständlich zu sein, einen Körper zu haben. Genau besehen sind sie aber, wie der Wasserfall, Prozess. Sie sind ein Ganzes, welches aus einer enorm grossen Zahl von ineinander verschachtelten Teilprozessen gebildet wird. Eine fast unendliche Zahl von biochemischen Vorgängen bildet eine Zelle; circa $10^{14}$ Zellen formen in unglaublich komplexer und feiner Abstimmung zueinander die Organe; letztere wiederum dienen als Basis für die Aktivitäten des Gesamtorganismus. ${ }^{2}$ Dabei lassen sich schnelle (Atmung, Herzkreislauf etc.) von langsam ablaufenden Prozessen (Knochen, ZNS) unterscheiden. Der Flusscharakter von Letzteren lässt sich daran erkennen, dass bei ihnen innert weniger Monate die Mehrzahl aller Moleküle entfernt und durch neue ersetzt werden.

Der Mensch, verstanden als ein komplexes Prozessgeschehen, schliesst sich der langen Denktradition an, die mit Heraklit begann und in dessen Dictum, «alles fliesst», seinen Ausdruck findet.

Prozesse haben einen Anfang und ein Ende. Kommen sie an ihr Ende, steht deren Fluss für einen kurzen Moment still (= Zustand), bevor ein nächster Prozess beginnt. Ist beispielsweise ein Atemzyklus 
beendet, dauert es einen kleinen Moment, bis der nächste Atemzug anhebt.

3. Für alle Prozesse gilt:

a. dass sie auf ein Ziel hin ausgerichtet sind. Mit «Ziel» ist ein möglicher (d.h. ein potentieller) zukünftiger Zustand gemeint. Es handelt sich nicht um eine aktuelle Realität, sondern um etwas Potentielles, möglicherweise nie aktuell Werdendes. Ziele sind im weitesten Sinne geistige Gebilde.

b. dass sie ihr Ziel sehr energisch verfolgen. Das «Streben nach» oder «sich Sehnen nach» der Lebewesen (der Prozesse) entspricht einer subjektiven Gestimmtheit und ist Quelle allen Werdens und Fliessens. Prozesse kommen nicht ohne diese aus dem subjektiven Erleben der Lebewesen stammende alles bewegende Energie aus.

c. dass sie ihren Anfang dort haben, wo vorangehende Prozesse ihr Ziel erreicht haben und zu einem Ende gekommen sind. Prozesse sind damit in die Gegebenheiten, welche aus früheren Prozessen resultieren, eingebettet. ${ }^{3}$

4. Prozesse gelangen nicht immer an ihr Ziel, sondern können auf ihrem Weg gestört oder vorzeitig gestoppt werden. Der Atem eines Menschen stockt bei plötzlich drohender Gefahr. Typischerweise führen Versuche, Prozesse zu dokumentieren oder zu messen, auch dazu, dass sie in ihrem Werden gestört oder gar zerstört werden. Der Versuch, besonders schöne und glückliche Momente - Ferienerlebnisse beispielsweise - mittels Fotos zu dokumentieren, unterbricht das Erlebnis, und zurück bleibt ein Artefakt des gelebten Lebens in Form einer Fotografie. Weil Fotografien oder Bilder als statische, zweidimensionale Artefakte wenig geeignet sind, Menschen adäquat zur Darstellung zu bringen, ist in diesem Artikel nicht von "Menschenbild», sondern von einer "Theorie des Menschen" die Rede. In Letztere lassen sich dynamische Vorstellungen und Ideen besser unterbringen.

Die Medizin ist zu einer Grossproduzentin von Bildern und anderen Artefakten geworden. Die radiologischen Bildgebungen, Endoskopien, EKG-, EEG-, Lungenfunktionskurven, Laborwerte etc. sind Artefakte, welche das Leben der erkrankten Person nur sehr teilweise zu erfassen vermögen. Gegen deren Produktion ist nichts einzuwenden, wenn klar bleibt, dass sie den Lebensvorgängen äusserlich bleiben und solange sie die Sicht auf Wesentliches in der Medizin, insbesondere den Umgang mit Emotionen und die Auseinandersetzung mit der condition humaine (d.h. mit Leiden und Tod), nicht verstellen.

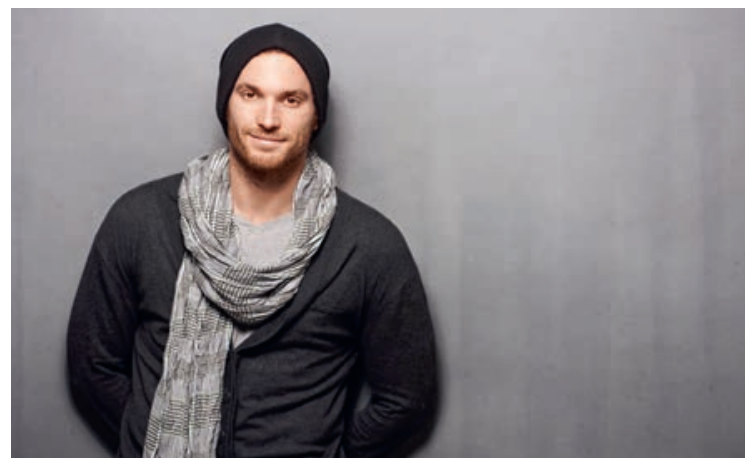

Abbildung 1: Artefakte - «ceci n'est pas un homme».

5. Prozesse laufen auf mikroskopischer und makroskopischer Ebene einzelner Lebewesen ab. Lebewesen lassen sich zudem in Prozesse ein, welche mehrere Individuen miteinander verbinden. Prozesshafte Vorgänge dieser Art stellen Gespräche zwischen zwei oder mehreren Menschen, gemeinsames Musizieren oder der Liebesakt dar. Das Ausmass dieses eingebunden Seins ist stärker als gemeinhin angenommen und geht über das Visuelle (den anderen sehen) und das Auditive (die Worte des anderen hören) hinaus. Innerhalb gemeinsamer Prozesse werden die lebenswichtigen emotionalen Beziehungen zu den Eltern und anderen Bezugspersonen aufgebaut. In der Medizin entstehen so therapeutische Allianzen und geheimnisvolle, physikalistisch-mechanistisch nicht erklärbare Phänomene, wie beispielsweise der Placebo-Effekt. Der Placebo-Effekt ist so gesehen Ausdruck eines prozesshaften Geschehens zwischen Ärztin oder Therapeut und Patient bzw. Patientin.

\section{Geschichten erzählen}

Die Sprachfähigkeit, die Aristoteles als Unterscheidungskriterium zwischen Mensch und Tier stark machte, entspricht nicht mehr dem gegenwärtigen Wissensstand: Heute ist klar, dass Tiersprachen weit verbreitet sind und von uns teilweise auch verstanden werden können. Als Unterscheidungskriterium zwischen Mensch und Tier ist die Sprache nicht geeignet. Wovon sich der Mensch vom Tier allerdings in entscheidender Weise abhebt, ist die Virtuosität in der Benützung von Sprache. Menschen sind fähig, mit Hilfe der Sprache nicht nur einzelne Hinweise («Achtung Löwe!», «Hau ab!» o.Ä.) zu geben. Sie können mit deren Hilfe neue Welten in Form von Geschichten schaffen und sich darin selber zum Gegenstand machen. Nach heutigem Stand des Wissens sind Menschen in ihrem Sprachgebrauch als Einzige selbstreflexiv. 
Die Fähigkeit, Geschichten zu erzählen, stellt nicht nur ein Unterscheidungsmerkmal zwischen Mensch und Tier dar. Geschichten haben auch die Entwicklung der Menschheit als Ganze geprägt. Man denke an die Bibel, den Koran, an Schöpfungsmythen, die Unabhängigkeitserklärung der Vereinigten Staaten, an die Bundesverfassung. Auch auf der individuellen Ebene kann der Einfluss von Geschichten kaum überschätzt werden. Man denke an die Familiengeschichte, die eigene Biographie oder Krankengeschichte; sie alle sind ganz wichtige Elemente im Leben jeder Person. Eng verbunden mit dem Erzählen von Geschichten steht das Gespräch. Darin ist die prozesshafte Verbundenheit bzw. Verschränkung der Gesprächspartner besonders intensiv.

Das Erzählen bzw. das Anhören von Geschichten der Patienten steht ausserdem im Zentrum der ärztlichen Sprechstunde und der ärztlichen Tätigkeit überhaupt. In den letzten Jahrzehnten wurde diesem Element ärztlicher Praxis ausserhalb der Psychiatrie nur noch wenig Beachtung geschenkt. Es gibt aber Anzeichen einer Rückbesinnung. Dies findet seinen Ausdruck darin, dass vermehrt von der sog. "narrativen» oder der «sprechenden» Medizin die Rede ist. ${ }^{4}$

\section{Theoretische und lebensweltliche Menschenbilder}

Die Medizin ist ein praxisorientiertes Fach. Entsprechend stehen ärztlich Tätige Theorien, wie sie soeben dargelegt wurden, skeptisch gegenüber. Sie fragen nach dem praktischen Nutzen und monieren, dass Theorien als abstrakte geistige Gebilde dem eigentlichen Leben fremd und wenig dienlich seien.

Es ist zu bedenken, dass jeder Praxis, sei sie noch so einfach und «handfest», eine Theorie zugrunde liegt. So verbringen beispielsweise Medizinstudenten die ersten Jahre ihrer Ausbildung mit dem Erlernen von physikalischen und biochemischen Theorien.

Natürlich sind Theorien über den Menschen abstrakter Natur, natürlich sind sie als Folge grundsätzlicher Überlegungen und alleine für sich genommen inhaltsleer, gar lebensfremd, und natürlich macht jeder Mensch im Laufe seines Lebens seine je persönlichen Erfahrungen im Umgang mit den Mitmenschen. Einige dieser Erfahrungen prägen sich aber besonders nachhaltig ein und geben die Basis für eine aus der Praxis erwachsende, individuelle Theorie vom Menschen ab. M. Zichy nennt es das «lebensweltliche Menschenbild».

Wenn ich hier eine allgemeine Theorie des Menschen bekannt mache, geschieht dies in der Hoffnung, dass sie, das lebensweltliche Menschenbild der Ärzte und Ärztinnen so beeinflussen und prägen wird, dass sie in ihrem Alltag die (Kranken-)Geschichten zwar individu- ell werten, dass sie diese aber in einer Theorie verorten, worin Menschen nicht mehr als mechanistisch, sondern prozess-theoretisch verstanden werden.

\section{Folgerungen}

Das Dargelegte, sollte es ernst genommen werden, lässt in der Medizin Änderungen und Anpassungen erwarten. Einige davon sollen nun genannt werden:

1. Änderung der Begrifflichkeit: Den Lesern wird aufgefallen sein, dass verschiedene Elemente des Maschinen- und des biospsychosozialen Modells des Menschen bei der PpT fehlen. Das gilt insbesondere für den «menschlichen Körper», wo es gilt, den Körper toter Gegenstände begrifflich strikte vom Menschen und Lebewesen generell zu trennen. Andere gängige Begriffe der heutigen Medizin, wie beispielsweise derjenige der «Seele», der «Psychosomatik» oder des "Gesundheitszustandes» müssten überdacht und neu bestimmt werden. Das ist allerdings schneller gesagt als getan, denn die Gewohnheit sitzt tief. In einem ersten Schritt wären sprachliche und gedankliche Alternativen auszuarbeiten und bekannt zu machen.

2. Änderung der Ausbildungsschwerpunkte für angehende Ärzte: Weil technische Eingriffe und maschinell hergestellte Artefakte (Bilder, Kurven, Messresultate u.a.m.) Lebensprozesse nicht oder nur sehr partiell zu erfassen vermögen, wird deren Stellenwert in der prozess-philosophisch orientierten Medizin herabgestuft werden. Um technische Eingriffe durchzuführen, ist die lange medizinische Ausbildung entbehrlich. Dafür wird die Kunst, zuzuhören, ein therapeutisches Gespräch zu führen, einen fachgerechten Status zu erheben oder in den häufig komplexen medizinischen Situationen erfolgreiche Wege zu weisen, finanziell besser abgegolten, gefördert und vermehrt ins Zentrum der ärztlichen Ausbildung gestellt werden.

3. Andere Methodik in der medizinischen Forschung: Messdispositive stören oder zerstören Prozesse, so dass es in der Erforschung von Krankheits- und Gesundungsvorgängen nötig sein wird, neue Forschungsmethoden zu entwickeln.

4. Neuausrichtung des Ziels der Medizin: Im Rahmen der PpT des Menschen besteht das Ziel ärztlicher Tätigkeit nicht in einer Reparaturarbeit, sondern vielmehr darin, wie ein Katalysator zu wirken: sich in einen gemeinsamen Entwicklungsprozess einlassen, welcher es dem Patienten ermöglicht, zu einem Leben zurückzufinden, welches für ihn Sinn macht.

Bildnachweis

(c) Nyul | Dreamstime.com 